Psychother Psychosom 2017;86:111-112

DOI: $10.1159 / 000453400$

\section{Restructuring Hedonic Dysregulation in Chronic Pain and Prescription Opioid Misuse: Effects of Mindfulness-Oriented Recovery Enhancement on Responsiveness to Drug Cues and Natural Rewards}

\author{
Eric L. Garland ${ }^{\mathrm{a}}$, Matthew O. Howard ${ }^{\mathrm{b}}$, Jon-Kar Zubieta ${ }^{\mathrm{a}}$, \\ Brett Froeliger ${ }^{c}$, \\ a University of Utah, Salt Lake City, UT, 'b University of North \\ Carolina at Chapel Hill, Chapel Hill, NC, and ${ }^{\mathrm{C}}$ Medical University \\ of South Carolina, Charleston, SC, USA
}

The allostatic model posits that chronic drug use induces hedonic homeostatic dysregulation, in which motivation to obtain natural rewards (e.g., eating, copulation, affiliation) is reorganized around seeking drug-induced reward to alleviate dysphoria [1]. The downward shift in salience of natural reward relative to drug reward may represent a crucial tipping point leading to the loss of control over drug use that is characteristic of addiction. Heightened responsiveness to drug reward coupled with decreased responsiveness to natural reward has been observed in opioid-dependent individuals $[2,3]$, predicts opioid consumption [4], and may drive prescription opioid misuse and addiction [5]. Therapies that restructure reward responsiveness from valuation of drug reward to valuation of natural reward may be effective means of treating opioid misuse.

We conducted an RCT of a Mindfulness-Oriented Recovery Enhancement (MORE) intervention for opioid misuse among chronic pain patients. MORE integrates skills to amplify natural reward processing with mindfulness and reappraisal techniques. In this RCT, relative to a support group (SG) control, MORE reduced opioid misuse and craving while decreasing pain symptoms [6]. Given its focus on orienting attention away from drug-related cues and towards healthful and socially affiliative objects and events, it is possible that MORE may regulate attention to hedonically relevant stimuli to shift the relative salience of drug and natural rewards, and thereby ameliorate opioid misuse.

To test this exploratory hypothesis, we examined unpublished psychophysiological data from this trial (ClinicalTrials.gov identifier NCT01505101) [6]. Individuals with complete data (17 men and 34 women, mean age $=45.7$ years, $\mathrm{SD}=13.7, \operatorname{MORE} n=20$; SG $n=31$ ) from an affective picture-viewing paradigm conducted 1 week before and after the study treatments were selected for the present analysis. Chronic pain patients were recruited from primary and specialty care clinics and met the inclusion criteria if they took opioids nearly every day for $>90$ days. Most (84.3\%) reported

\section{KARGER}

(c) 2017 S. Karger AG, Basel

E-Mail karger@karger.com

www.karger.com/pps opioid misuse as defined by a validated cutoff point on the Current Opioid Misuse Measure (COMM >12) [7]. We used heart rate (HR) to measure cue responsiveness and high-frequency heart rate variability (HRV) to index parasympathetic regulation of hedonic responses, including attention to emotional information [8]. Participants were paid USD 200 for completing the IRB-approved study; all procedures complied with the Helsinki Declaration.

The manualized 8-session MORE treatment involved group training in mindfulness to disengage attention from drug cues and intentionally reorient attention to visual, auditory, olfactory, gustatory, or tactile features of a pleasant experience (e.g., the warmth of the sun on one's skin) while cultivating metacognitive awareness of positive emotions and cognitions arising in response to the pleasant event [9]. The 2-h sessions were led by a social worker and supervised weekly by the developer of MORE. Participants engaged in daily 15-min mindfulness sessions at home guided by a CD. The manualized SG control condition consisted of 8 weekly, 2-h sessions, in which a social worker facilitated emotional expression and discussion of chronic pain-related topics. MORE and SG session recordings were reviewed to maintain treatment fidelity.

Participants performed a randomized, event-related affective picture-viewing task [10] in which they passively viewed neutral (furniture, neutral faces), pain-related (injuries, medical proce-

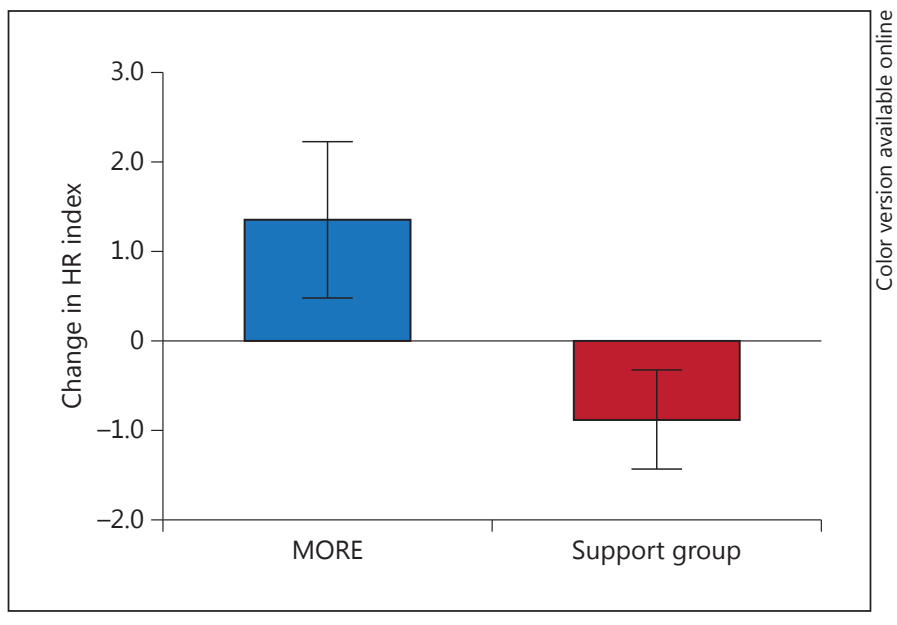

Fig. 1. Changes in relative responsiveness to natural reward and opioid cues from pre- to postintervention $(n=51)$. Positive scores indicate increased natural reward cue-elicited $\mathrm{HR}$ relative to opioid cue-elicited HR. Negative scores indicate decreased natural reward cue-elicited HR relative to opioid cue-elicited HR. The group $\times$ time effect was significant $\left(F(1,49)=7.61, p=0.008, \eta_{\text {partial }}^{2}=\right.$ 0.13). MORE, Mindfulness-Oriented Recovery Enhancement; $\mathrm{HR}$, heart rate. 
dures), natural reward (social attachment, nature scenes), and opioid-related cues (pills, pill bottles). After a 500-ms fixation cross, pictures were presented for $6 \mathrm{~s}$ while HR was recorded, after which stimulus valence and arousal were rated on 9-point Likert scales. $\mathrm{HR}$ was recorded at $1,000 \mathrm{~Hz}$ on a Biopac MP150. Kubios $2.0 \mathrm{soft}-$ ware computed time domain HRV analyses, yielding the root mean square of successive differences to estimate parasympathetically mediated HRV. Cue-elicited HRV responsivity scores were generated by covarying HRV during a 5-min baseline from HRV during affective picture viewing. Cue-elicited $\mathrm{HR}$ was averaged for each cue type.

Repeated-measures ANOVA revealed a significant group (MORE vs. SG) $\times$ time (pre vs. posttreatment) effect on HRV responsivity $\left(F(1,49)=4.42, p=0.04, \eta^{2}\right.$ partial $\left.=0.08\right)$, indicating that compared to the SG the MORE group experienced significantly greater increases in HRV responsivity during affective picture viewing. Repeated-measures ANOVA was conducted on cue-elicited HR with group, time, and cue type (opioid, natural reward, pain, and neutral) as factors. We identified a significant group $\times$ time $\times$ condition effect $\left(F(3,47)=4.54, p=0.007, \eta_{\text {partial }}^{2}=0.09\right)$, indicating that compared to the SG the MORE group experienced significantly greater decreases in cue-elicited HR. This effect was most pronounced for drug cue-elicited HR relative to natural reward cue-elicited $\operatorname{HR}\left(F(1,49)=7.59, p=0.004, \eta_{\text {partial }}^{2}=0.13\right)$.

We computed a measure of relative responsiveness to natural reward cues compared to drug cues by extracting residuals generated from regression analyses in which drug cue-elicited HR was regressed on natural reward cue-elicited HR. Repeated-measures ANOVA revealed a significant group $\times$ time effect on this relative responsiveness measure $\left(F(1,49)=7.61, p=0.008, \eta^{2}\right.$ partial $\left.=0.13\right)$, indicating that compared to the SG the MORE group experienced significantly greater increases in responsiveness to natural reward cues relative to drug cues from pre- to posttreatment (see Fig. 1). Controlling for pretreatment opioid misuse severity, the residualized change in relative responsiveness significantly predicted COMM opioid misuse scores at the 3-month follow-up: $B=-0.32$, $p<0.05$ (model $R^{2}=0.32$ ). Compared to the SG, MORE was associated with significantly higher posttreatment arousal ratings of natural reward cues $\left(F(1,49)=9.30, p=0.004, \eta_{\text {partial }}^{2}=0.21\right)$.

These preliminary findings tentatively suggest that MORE may enhance the autonomic regulation of perturbations by hedonic stimuli, and in so doing modulate the relative salience of natural reward and drug-related cues to reduce opioid misuse. MORE is a sequenced treatment designed to modify associative learning mechanisms by strengthening top-down cognitive control to restructure bottom-up reward learning from valuation of drug reward to valuation of natural reward. It is possible that this restructuring of reward may arise from the restoration of prefrontalstriatal feedback [9], but more well-controlled mechanistic re- search is needed to test this neural hypothesis. Insofar as addiction involves a downward shift in salience of natural reward relative to drug reward, interventions that reverse this allostatic process by restructuring reward learning may prove to be highly efficacious.

\section{Acknowledgments}

This work was supported by grants from the National Institutes of Health (NIH) awarded to E.L.G. (grant No. R03DA032517 and R01DA042033) and a grant from the Fahs Beck Fund for Research and Experimentation, also awarded to E.L.G. The conclusions in this article are those of the authors and do not necessarily represent the official position of the NIH. The authors have no conflict of interest to report with regard to the contents of this manuscript. E.L.G. has conducted MORE training for which he received monetary incentives.

\section{References}

1 Koob GF, Le Moal M: Drug addiction, dysregulation of reward, and allostasis. Neuropsychopharmacology 2001;24:97-129.

2 Lubman DI, Allen NB, Peters LA, Deakin JF: Electrophysiological evidence that drug cues have greater salience than other affective stimuli in opiate addiction. J Psychopharmacol 2008;22:836-842.

3 Garland EL, Froeliger B, Howard MO: Allostatic dysregulation of natural reward processing in prescription opioid misuse: autonomic and attentional evidence. Biol Psychol 2015;105:124-129.

4 Lubman DI, Yucel M, Kettle JW, Scaffidi A, Mackenzie T, Simmons JG et al: Responsiveness to drug cues and natural rewards in opiate addiction: associations with later heroin use. Arch Gen Psychiatry 2009;66: 205-212.

5 Garland EL, Froeliger B, Zeidan F, Partin K, Howard MO: The downward spiral of chronic pain, prescription opioid misuse, and addiction: cognitive, affective, and neuropsychopharmacologic pathways. Neurosci Biobehav Rev 2013;37:2597-2607.

6 Garland EL, Manusov EG, Froeliger B, Kelly A, Williams JM, Howard MO: Mindfulness-oriented recovery enhancement for chronic pain and prescription opioid misuse: results from an early-stage randomized controlled trial. J Consult Clin Psychol 2014;82:448-459.

7 Butler SF, Budman SH, Fernandez KC, Houle B, Benoit C, Katz N, et al: Development and validation of the Current Opioid Misuse Measure. Pain 2007;130:144-156.

8 Thayer JF, Lane RD: Claude Bernard and the heart-brain connection: further elaboration of a model of neurovisceral integration. Neurosci Biobehav Rev 2009;33:81-88.

9 Garland EL: Restructuring reward processing with Mindfulness-Oriented Recovery Enhancement: novel therapeutic mechanisms to remediate hedonic dysregulation in addiction, stress, and pain. Ann NY Acad Sci 2016;1373:25-37.

10 Cuthbert BN, Schupp HT, Bradley MM, Birbaumer N, Lang PJ: Brain potentials in affective picture processing: covariation with autonomic arousal and affective report. Biol Psychol 2000;52:95-111. 